\title{
Psocoptera (Insecta: Psocodea) from the National Natural Park Gorgona, Cauca, Colombia
}

\author{
Fabio Sarria-S. ${ }^{*}$, Ranulfo González O. ${ }^{1}$ \& Alfonso Neri García Aldrete ${ }^{2}$ \\ 1. Departamento de Biología, Facultad de Ciencias Naturales y Exactas, Universidad del Valle, Calle 13, 100-00, Fax \\ 3393243, tel. 3212100 ext 3137, Santiago de Cali, Colombia; fabsarria@gmail.com; \\ ranulfo.gonzalez@correounivalle.edu.co \\ 2. Departamento de Zoología, Instituto de Biología, Universidad Nacional Autónoma de México. Apartado Postal \\ 70-153, 04510 México, D. F., México; anga@ibunam2.ibiologia.unam.mx
}

\author{
Recibido 18-X-2013. Corregido 20-XI-2013. Aceptado 19-XII-2013.
}

\begin{abstract}
The Psocoptera fauna of Gorgona National Natural Park, Colombian pacific, consists of 75 species in 42 genera and 21 families. 1730 specimens were collected in the period November 2007-June 2011. Five families, 20 genera and nine species are new records for Colombia, and two genera and ten species are new to science. The psocid fauna of the island constitutes an extension of the continental fauna. Rev. Biol. Trop. 62 (Suppl. 1): 243-256. Epub 2014 February 01.
\end{abstract}

Key words: Pacific coast, Colombia, natural national parks, species list.

Psocids (Psocodea: 'Psocoptera'), are small, neopterous, exopterygote insects, characterized by having filiform antennae, bulbous postclypeus, asymmetric mandibles, and maxillae with elongate, free laciniae; the wings are membranous, with simple venation, in most species placed roof-like over the dorsum; apterism and brachypterism are frequent, and the tarsi are 2-3 segmented in the adults and always 2 segmented in the nymphs. For general information on the biology of these insects, see Badonnel (1951), Lienhard (1998), Mockford (1993) and New (1987). For relationships within the order Psocodea, see Bess, Smith, Lienhard \& Johnson (2006). They are essentially wild, free living insects that constitute part of the aerial plankton (Simberloff \& Wilson, 1969), and represent a significant fraction of the biomass in temperate forests (Thornton, 1985); they are probably key organisms in the trophic network of many ecosystems, as they are prey of many vertebrates and invertebrates, and they are primary consumers, feeding on algae, lichens, mycelia that grow on foliage, tree trunks and rock surfaces, and occasionally on pollen grains (Lienhard, 1998). They are found in living and dead foliage, leaf litter, cortex of trees and shrubs, rock surfaces and human habitations (Mockford, in prep., GarcíaAldrete, 1990).

It is a diverse group, with more than 5500 species, in more than 435 valid genera, and more than 40 families (García-Aldrete, 2006). They have been found all over the world, being particularly species rich in the tropics (GarcíaAldrete, 1988). In Colombia, only a few species have been recorded so far (87 species), but being a megadiverse country, we anticipate that its psocid richness will be comparable to the richness of other neotropical countries, although for most of them the inventory is rather incomplete (see García-Aldrete, 2008, for a list of Mexican Psocoptera, and García-Aldrete \& Mockford, 2009, for a list of Brazilian Psocoptera). The Pacific islands of Gorgona and Gorgonilla presently constitute the National 
Natural Park (NNP) Gorgona, a protected area in Colombia that was strongly altered anthropogenically in the past, on account of the establishment there of a high security prison, cancelled in 1984. It constitutes a representative area of highly humid tropical forest in the biogeographic Colombian Chocó, quite diverse biologically. The arthropods in general have been little studied, and in this work we present a preliminary list of Psocoptera, with information on the habitats where they have been found, resulting from field work in the NNP Gorgona, between 2007 and 2011.

\section{MATERIAL AND METHODS}

The NNP Gorgona belongs to the municipality of Guapi $\left(2^{\circ} 47^{\prime}-3^{\circ} 6^{\prime} \mathrm{N}, 78^{\circ} 6^{\prime}-78^{\circ} 18^{\prime}\right.$ W), Department of Cauca, in the south of the Colombian Pacific; it includes the islands of Gorgona and Gorgonilla, with an area of $13.8 \mathrm{Km}^{2}$ and with altitudes from 0 to $338 \mathrm{~m}$ at Cerro Trinidad (Chamorro, 1990). It presents, as average annuals: temperature $26^{\circ} \mathrm{C}$, relative humidity $90 \%$, solar brightness $989.5 \mathrm{~h}$, precipitation $6891.4 \mathrm{~mm}$ and evaporation $900.2 \mathrm{~mm}$ (Rangel \& Rudas, 1990).

The specimens were taken directly from the substrate, by beating vegetation, by sweeping with a soft brush (3-4 inches wide), tree trunks and rock surfaces, and by sifting leaf litter; the specimens were taken in beating cloths of about $60 \times 60 \mathrm{~cm}$, or in plastic, rectangular trays $(25 \times 40 \times 10 \mathrm{~cm})$, and transferred to containers with $80 \%$ ethyl alcohol, utilizing mouth aspirators. Malaise, Shannon and led light traps were also utilized, placing the latter near the canopy, at heights of 15 and 20m. For the identification, it was necessary to dissect the specimens and mount their parts permanently on slides in Canada balsam (see García-Aldrete, 1990 and González-Obando, García-Aldrete $\&$ Carrejo, 2011). Table 1 lists the coordinates, altitudes and abbreviations for the collecting localities (Fig. 1) noted for each species.

\section{RESULTS}

1730 specimens were taken, in which 75 species of Psocoptera are represented, in 42 genera and 21 families; six families, 21 genera and 20 species constitute new records for the Colombian fauna, at least ten species are new to science, three of which have just been recently described (García-Aldrete, Gonzalez \& Sarria-S 2011). The most diverse family is Psocidae, with eight genera and 18 species, followed by Lepidopsocidae, with five genera and six species; the most speciose genera are Ectopsocus (eight species), Valenzuela (six species), and Blastopsocus (five species); six genera are represented by two species, and 25 genera are represented by one species. Table 2 summarizes densities and the different habitats where the species were collected.

\section{Species List:}

Suborder Trogiomorpha

Family Lepidopsocidae

\section{Echmepteryx falco (Badonnel, 1949)}

Records: A1, A4, A8, A11, A13, A14, A15, A16, A17, 12-17.XI.2007, canopy fogging, 30 우. S.PB, 25.XI.2009, beating foliage, 2 우아. S.PB, 25.XI.2009, in leaf litter, 3 우. S.FGll, 26.XI.2009, beating foliage, 2 우우. Pd, 24.XI.2009, beating foliage, 91 우우. S.Ch, 21.XI.2009, beating foliage, 6 우. PGll, 26.XI.2009, on palm trunk and foliage, 12 우우. PB, 19.X.2010, beating foliage, 17 우우. S.PB, 19.X.2010, beating foliage, 1 ․ PGll, 16.X.2010, beating foliage, 12 ㅇ․ PP, 17.X. 2010, beating foliage, 17 우. Pd, 22.X.2010, beating foliage, 1 ․ S.Ch, 18.X.2010, beating foliage, 10 웅. S.Ch, 23.X.2010, beating foliage, 7 우. PP, 24.II.2011, beating foliage, 3 우. S.PB, 26.II.2011, beating foliage, 5 우. PGll, 22.II. 2011, beating foliage, 5 우오. PCa, 23.II.2011, beating foliage, 5 웅. 


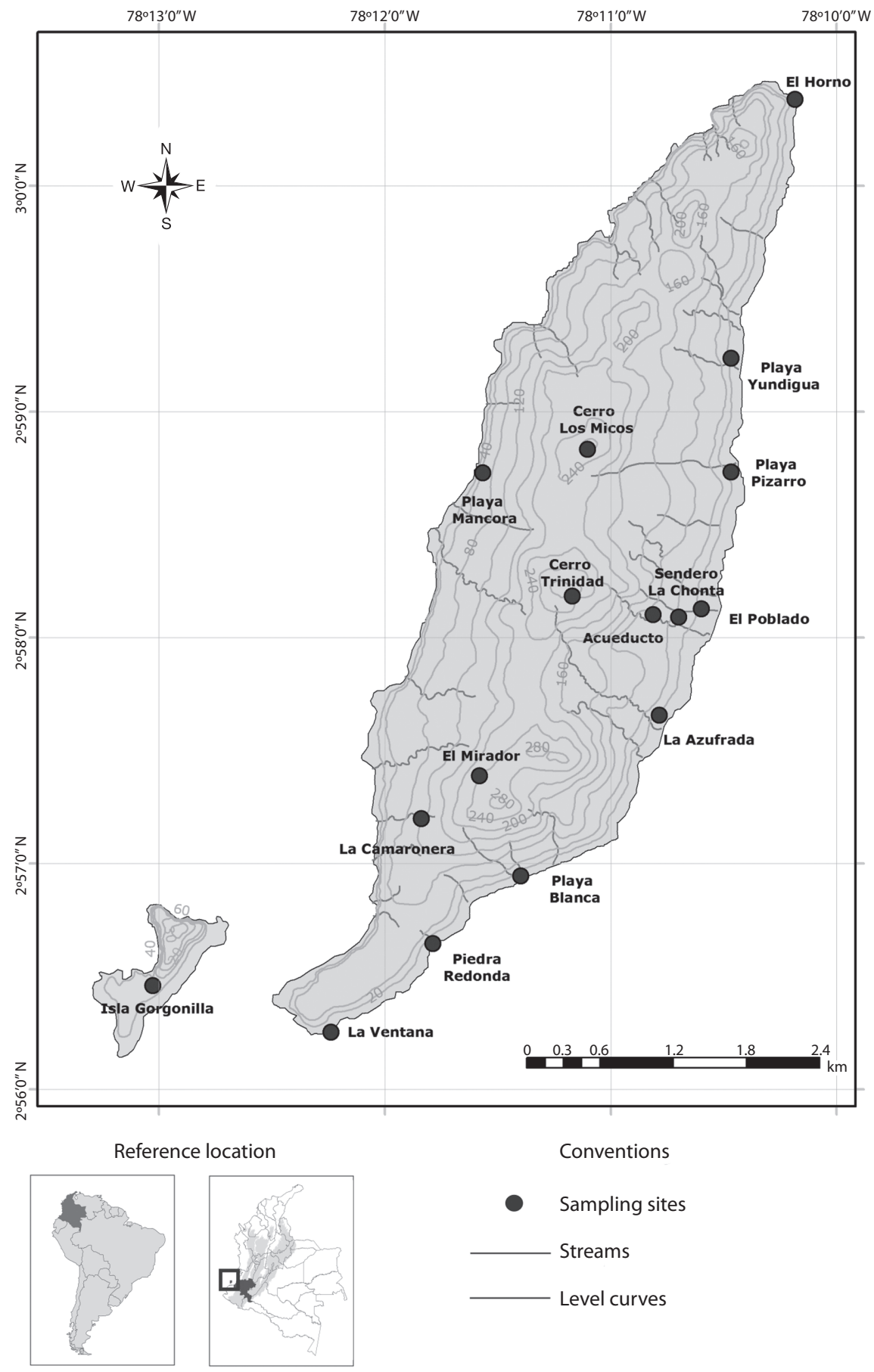

Fig. 1. Sampling locations in PNN Gorgona. Paths between locations are not indicated 
TABLE 1

Keys and coordinates of collecting localities in the NNP Gorgona

\begin{tabular}{|c|c|c|c|}
\hline Key & Description & Coordinates & Meters (asl) \\
\hline A1 & Tree \#1 & $02^{\circ} 57^{\prime} 51.8^{\prime \prime} \mathrm{N}-78^{\circ} 10^{\prime} 25.9^{\prime \prime} \mathrm{W}$ & 7 \\
\hline A2 & Tree \#2 & $02^{\circ} 57^{\prime} 53.9^{\prime \prime} \mathrm{N}-78^{\circ} 10^{\prime} 32.1 ” \mathrm{~W}$ & 16 \\
\hline A3 & Tree \#3 & $02^{\circ} 57^{\prime} 53.5^{\prime \prime} \mathrm{N}-78^{\circ} 10^{\prime} 32.2^{\prime \prime} \mathrm{W}$ & 16 \\
\hline A4 & Tree \#4 & $02^{\circ} 57^{\prime} 53.6^{\prime \prime} \mathrm{N}-78^{\circ} 10^{\prime} 32.0^{\prime \prime} \mathrm{W}$ & 3 \\
\hline A5 & Tree \#5 & $02^{\circ} 57^{\prime} 56.2^{\prime \prime} \mathrm{N}-78^{\circ} 10^{\prime} 31.2^{\prime \prime} \mathrm{W}$ & 3 \\
\hline A6 & Tree \#6 & $02^{\circ} 57^{\prime} 56.2^{\prime \prime} \mathrm{N}-78^{\circ} 10^{\prime} 31.2^{\prime \prime} \mathrm{W}$ & 3 \\
\hline A7 & Tree \#7 & $02^{\circ} 57^{\prime} 56.2^{\prime \prime} \mathrm{N}-78^{\circ} 10^{\prime} 31.2^{\prime \prime} \mathrm{W}$ & 3 \\
\hline A 8 & Tree \#8 & $02^{\circ} 57^{\prime} 55.9^{\prime \prime} \mathrm{N}-78^{\circ} 10^{\prime} 27.6^{\prime \prime} \mathrm{W}$ & 2 \\
\hline A9 & Tree \#9 & $02^{\circ} 57^{\prime} 54.4^{\prime \prime} \mathrm{N}-78^{\circ} 10^{\prime} 25.8^{\prime \prime} \mathrm{W}$ & 2 \\
\hline A10 & Tree \#10 & $02^{\circ} 57^{\prime} 54.4^{\prime \prime} \mathrm{N}-78^{\circ} 10^{\prime} 25.8^{\prime \prime} \mathrm{W}$ & 2 \\
\hline A11 & Tree \#11 & $02^{\circ} 57^{\prime} 54.4^{\prime \prime} \mathrm{N}-78^{\circ} 10^{\prime} 25.8^{\prime \prime} \mathrm{W}$ & 2 \\
\hline A12 & Tree\#12 & $02^{\circ} 57^{\prime} 54.4^{\prime \prime} \mathrm{N}-78^{\circ} 10^{\prime} 25.8^{\prime \prime} \mathrm{W}$ & 2 \\
\hline A13 & Tree \#13 & $02^{\circ} 58^{\prime} 11.8^{\prime \prime} \mathrm{N}-78^{\circ} 10^{\prime} 31.5^{\prime \prime} \mathrm{W}$ & 123 \\
\hline A14 & Tree \#14 & $02^{\circ} 58^{\prime} 12.3^{\prime \prime} \mathrm{N}-78^{\circ} 10^{\prime} 32.0^{\prime \prime} \mathrm{W}$ & 117 \\
\hline A 15 & Tree \#15 & $02^{\circ} 58^{\prime} 13.9^{\prime \prime} \mathrm{N}-78^{\circ} 10^{\prime} 34.8^{\prime \prime} \mathrm{W}$ & 123 \\
\hline A16 & Tree \#16 & $02^{\circ} 58^{\prime} 13.3^{\prime \prime} \mathrm{N}-78^{\circ} 10^{\prime} 34.6^{\prime \prime} \mathrm{W}$ & 119 \\
\hline Acd & Sendero del Acueducto & $02^{\circ} 57^{\prime} 11.9^{\prime \prime} \mathrm{N}-78^{\circ} 10^{\prime} 13.5^{\prime \prime} \mathrm{W}$ & 100 \\
\hline $\mathrm{CM}$ & Cerro de los Micos & $02^{\circ} 58^{\prime} 06.4^{\prime \prime} \mathrm{N}-78^{\circ} 11^{\prime} 33.0^{\prime \prime} \mathrm{W}$ & 246 \\
\hline $\mathrm{CMr}$ & Cerro del Mirador & $02^{\circ} 57^{\prime} 09.0^{\prime \prime} \mathrm{N}-78^{\circ} 11^{\prime} 03.6^{\prime \prime} \mathrm{W}$ & 241 \\
\hline CT1 & Cerro Trinidad, site 1 & $02^{\circ} 58^{\prime} 20.3^{\prime \prime} \mathrm{N}-78^{\circ} 10^{\prime} 43.5^{\prime \prime} \mathrm{W}$ & 169 \\
\hline $\mathrm{CT} 2$ & Cerro Trinidad, site 2 & $02^{\circ} 58^{\prime} 13.4^{\prime \prime} \mathrm{N}-78^{\circ} 10^{\prime} 57.8^{\prime \prime} \mathrm{W}$ & 226 \\
\hline FGll & Faro Gorgonilla & $02^{\circ} 57^{\prime} 01.6^{\prime \prime} \mathrm{N}-78^{\circ} 11^{\prime} 15.2^{\prime \prime} \mathrm{W}$ & 13 \\
\hline PB & Playa Blanca & $02^{\circ} 56^{\prime} 52.7^{\prime \prime} \mathrm{N}-78^{\circ} 11^{\prime} 32.4^{\prime \prime} \mathrm{W}$ & 3 \\
\hline $\mathrm{PCa}$ & Playa la Camaronera & $02^{\circ} 57^{\prime} 13.3^{\prime \prime} \mathrm{N}-78^{\circ} 11^{\prime} 48.6^{\prime \prime} \mathrm{W}$ & 5 \\
\hline $\mathrm{Pd}$ & El Poblado & $02^{\circ} 57^{\prime} 57.7^{\prime \prime} \mathrm{N}-78^{\circ} 10^{\prime} 24.4^{\prime \prime} \mathrm{W}$ & 21 \\
\hline PGll & Playa Gorgonilla & $02^{\circ} 56^{\prime} 39.2^{\prime \prime} \mathrm{N}-78^{\circ} 12^{\prime} 45.0^{\prime \prime} \mathrm{W}$ & 4 \\
\hline $\mathrm{PH}$ & Playa El Horno & $03^{\circ} 00^{\prime} 03.0^{\prime \prime} \mathrm{N}-78^{\circ} 10^{\prime} 21.0^{\prime \prime} \mathrm{W}$ & 68 \\
\hline PP & Playa Palmeras & $02^{\circ} 56^{\prime} 38.0^{\prime \prime} \mathrm{N}-78^{\circ} 12^{\prime} 08.7^{\prime \prime} \mathrm{W}$ & 19 \\
\hline PR & Playa Piedra Redonda & $02^{\circ} 56^{\prime} 08.6^{\prime \prime} \mathrm{N}-78^{\circ} 11^{\prime} 09.4^{\prime \prime} \mathrm{W}$ & 32 \\
\hline PY & Playa Yundigua & $02^{\circ} 98^{\prime} 52.8^{\prime \prime} \mathrm{N}-78^{\circ} 17^{\prime} 05.5^{\prime \prime} \mathrm{W}$ & 7 \\
\hline QC & Quebrada Chorro del Cura & $02^{\circ} 58^{\prime} 02.3^{\prime \prime} \mathrm{N}-78^{\circ} 10^{\prime} 09.0^{\prime \prime} \mathrm{W}$ & 63 \\
\hline S. Ch & Trail El Poblado - Hydroelectric Microcentral- Interpretation Center & Approximately $1 \mathrm{Km}$ & N/A \\
\hline S. FGll & Trail Playa Gorgonilla-Faro. & Approximately $0.5 \mathrm{Km}$ & N/A \\
\hline S. PB & Trail Poblado-Playa Blanca & Approximately $3 \mathrm{Km}$ & \\
\hline S. PP & Trail Playa Redonda-Playa Palmeras & Approximately $1.3 \mathrm{Km}$ & 51 \\
\hline S. PY & Trail El Poblado - Playa Yundigua & Approximately $2.3 \mathrm{Km}$ & N/A \\
\hline
\end{tabular}

2. Echmepteryx madagascariensis (Kolbe, 1885)

Records: S.PB, 25.XI.2009, on dead leaves, 1 ․ PGll, 26.XI.2009, on palm trunk, 20 우. $\mathrm{Pd}$, 24.XI.2009, beating foliage, 4 우.PB, 19.X. 2010, beating foliage, 12 우. PGll, 16.X.2010, beating foliage, 87 웅. PP, 17.X.2010, beating foliage, 99 우. Pd, 22.X.2010, beating foliage, 1 ‥ S.Ch, 18.X.2010, beating foliage, 4 우우.
PGll, 22.II.2011, beating foliage, 6 우. PP, 22.II.2011, beating foliage, 2 웅, 2 ठㅎ․ P.Ca, 23.II.2011, beating foliage, 1 ․ PP, 24.II.2011, beating foliage, 18 우, $7 \hat{\jmath} \widehat{\jmath}$.

3. Lepidopsocus pretiosus (Banks, 1942)

Records: PGll, 26.XI.2009, beating foliage, 1 ․ S.Ch, 23.XI.2009, beating foliage, 1 ․ Pd, 24.XI.2009, beating foliage, 3 
TABLE 2

Species of Psocoptera and their habitats. $\mathrm{N}=$ number of individuals, $\mathrm{a}=$ foliage and branches, $\mathrm{b}=$ understory (15-20m, above ground), $\mathrm{c}=$ human edifices, $\mathrm{d}=$ tree trunks or under webbing, $\mathbf{e}=$ leaf litter; the asterisk $\left(^{*}\right)$ indicate the families, genera and species newly recorded in Colombia

\begin{tabular}{|c|c|c|c|c|c|}
\hline Species & $\mathrm{N}$ & Habitat & Species & $\mathrm{N}$ & Habitat \\
\hline LEPIDOPSOCIDAE & & & Ectopsocus sp. 6 & 1 & $\mathrm{~b}$ \\
\hline Echmepteryx falco* & 229 & $a, b, e$ & Ectopsocus sp. 7 & 1 & $\mathrm{~b}$ \\
\hline E. madagascariensis* & 264 & $\mathrm{a}, \mathrm{d}, \mathrm{e}$ & Ectopsocus sp. 8 & 2 & a \\
\hline Lepidopsocus pretiosus * & 17 & $\mathrm{a}$ & PERIPSOCIDAE & & \\
\hline Lepolepis sp. & 4 & $\mathrm{a}, \mathrm{e}$ & Peripsocus sp. 1 & 16 & $a, b$ \\
\hline Proentomum personatum * & 3 & a & Peripsocus sp. 2 & 10 & $\mathrm{~b}$ \\
\hline Nepticulomima hoesemanni* & 63 & $\mathrm{a}, \mathrm{c}$ & Peripsocus sp. 3 & 7 & $\mathrm{a}, \mathrm{b}$ \\
\hline AMPHIENTOMIDAE & & & Peripsocus sp. 4 & 4 & $a, b$ \\
\hline Seopsocus sp.* & 2 & $\mathrm{~b}$ & Kaestneriella ecuatoriana* & 8 & $\mathrm{a}, \mathrm{b}$ \\
\hline LIPOSCELIDIDAE & & & ca. Kaestneriella sp. (n. gen.) & 9 & $a, b$ \\
\hline Belaphotroctes sp. & 4 & d & ARCHIPSOCIDAE & & \\
\hline Liposcelis sp. & 8 & $\mathrm{~b}$ & Archipsocus sp. & 35 & $\mathrm{a}, \mathrm{b}, \mathrm{e}$ \\
\hline PACHYTROCTIDAE & & & Pararchipsocus sp.* & 29 & $\mathrm{a}$ \\
\hline Tapinella sp. & 3 & a & PSEUDOCAECILIIDAE & & \\
\hline EPIPSOCIDAE & & & Heterocaecilius sp.* & 123 & $a, b$ \\
\hline Goja sp. (n. sp.) & 18 & $\mathrm{a}, \mathrm{d}$ & Pseudocaecilius citricola & 12 & $a, b$ \\
\hline Mesepipsocus sp. (n.sp.)* & 19 & $\mathrm{a}, \mathrm{c}$ & Scytopsocus sp.* & 63 & $\mathrm{a}, \mathrm{b}$ \\
\hline Epipsocus sp. $1 *$ & 4 & $\mathrm{a}, \mathrm{e}$ & PHILOTARSIDAE & & \\
\hline Epipsocus sp. 2* & 2 & $\mathrm{~b}$ & Aaroniella sp. & 19 & $a, b$ \\
\hline PTILONEURIDAE & & & COMPSOCIDAE & & \\
\hline Loneura gorgonaensis & 3 & $\mathrm{~b}$ & Compsocus elegans* & 2 & $\mathrm{~b}, \mathrm{c}$ \\
\hline Loneura insularis & 6 & $\mathrm{~b}$ & HEMIPSOCIDAE* & & \\
\hline Loneura monticola & 6 & $\mathrm{~b}$ & Hemipsocus africanus * & 26 & a \\
\hline CLADIOPSOCIDAE* & & & PSOCIDAE & & \\
\hline Cladiopsocus sp.* & 16 & $a, b$ & Blaste sp. & 15 & $a, b$ \\
\hline DOLABELLOPSOCIDAE & & & Blastopsocus sp. 1 & 2 & $\mathrm{~b}$ \\
\hline Dolabellopsocus sp. & 8 & $\mathrm{a}, \mathrm{b}$ & Blastopsocus sp. 2 & 9 & $a, b$ \\
\hline Isthmopsocus n sp. & 21 & $\mathrm{a}, \mathrm{b}, \mathrm{e}$ & Blastopsocus sp. 3 & 13 & $\mathrm{a}, \mathrm{b}$ \\
\hline ASIOPSOCIDAE* & & & Blastopsocus sp. 4 & 4 & $\mathrm{a}, \mathrm{b}$ \\
\hline Notiopsocus sp. * & 1 & $\mathrm{~b}$ & Blastopsocus sp. 5 & 5 & $\mathrm{~b}$ \\
\hline CAECILIUSIDAE & & & Indiopsocus sp. 1 & 5 & $\mathrm{~b}$ \\
\hline Valenzuela sp. 1 & 11 & $\mathrm{~b}$ & Indiopsocus sp. 2 & 79 & $a, b$ \\
\hline Valenzuela sp. 2 & 74 & $\mathrm{a}$ & Indiopsocus sp. 3 & 1 & $\mathrm{a}$ \\
\hline Valenzuela sp. 3 & 51 & $\mathrm{a}, \mathrm{b}$ & Psococerastis sp. 1 & 11 & $\mathrm{a}, \mathrm{b}$ \\
\hline Valenzuela sp. 4 & 11 & $\mathrm{a}, \mathrm{b}, \mathrm{c}$ & Psococerastis sp. 2 & 6 & $\mathrm{~b}$ \\
\hline Valenzuela sp. 5 & 2 & $\mathrm{a}$ & Psococerastis sp. 3 & 7 & $\mathrm{a}, \mathrm{b}, \mathrm{e}$ \\
\hline Valenzuela sp. 6 & 7 & $a, b$ & Ptycta sp. $1^{*}$ & 10 & $\mathrm{~b}$ \\
\hline STENOPSOCIDAE* & & & Ptycta sp. 2* & 20 & $\mathrm{~b}$ \\
\hline Graphopsocus cruciatus* & 4 & $\mathrm{~b}$ & Thyrsopsocus sp. $1^{*}$ & 2 & $\mathrm{~b}$ \\
\hline LACHESILLIDAE & & & Thyrsopsocus sp. 2* & 3 & $\mathrm{a}, \mathrm{b}$ \\
\hline Lachesilla sp. 1 & 4 & $a, b$ & Trichadenotecnum sp.* & 2 & $\mathrm{~b}$ \\
\hline Lachesilla sp. 2 & 13 & $a, b$ & New Genus & 3 & $\mathrm{~b}$ \\
\hline ECTOPSOCIDAE & & & MYOPSOCIDAE* & & \\
\hline Ectopsocus sp. 1 & 18 & $\mathrm{a}$ & Myopsocus sp. 1* & 32 & $\mathrm{~b}$ \\
\hline Ectopsocus sp. 2 & 76 & $\mathrm{a}, \mathrm{d}$ & Myopsocus sp. 2* & 14 & $\mathrm{~b}$ \\
\hline Ectopsocus sp. 3 & 30 & $\mathrm{a}, \mathrm{d}$ & Lichenomima sp. 1* $^{*}$ & 33 & $\mathrm{a}, \mathrm{b}$ \\
\hline Ectopsocus sp. 4 & 13 & $\mathrm{a}$ & Lichenomima sp. $2 *$ & 55 & $\mathrm{~b}$ \\
\hline Ectopsocus sp. 5 & 16 & $\mathrm{a}, \mathrm{d}$ & Lichenomima sp. $3 *$ & 1 & $\mathrm{~b}$ \\
\hline
\end{tabular}


우. PP, 17.X.2010, beating foliage, 2 웅․ PB, 19.X.2010, beating foliage, 7 우. PP, 23.II.2011, light trap, 3 우.

\section{Lepolepis sp.}

Records: S.PB, 25.XI.2009, in leaf litter, 2 우. PP, 17.X.2010, beating foliage, 1 ․ PP, 24.II.2011, beating foliage, 1 ㅇ․

5. Proentomum personatum Badonnel, 1949

Records: S.Ch, 23.XI.2009, beating foliage, 1 ㅇ. S.Ch, 21.XI.2009, beating foliage, 1 ㅇ. PP, 24.II.2011, beating foliage, 1 ㅇ.

6. Nepticulomima hoesemanni (Enderlein, 1903)

Records: Pd, 26.XI.2009, on house wall, 4 우이 Pd, 24.XI.2009, beating foliage, 1 ․ Pd, 21.XI.2009, on window wood frame, 4 우으. Pd, 22.XI.2009, on wooden box, 34 우. Pd, 22.X.2010, on house wall, 13 우, 6 ठิ $\sigma^{\top}$. S.PY, 25.II.2011, beating foliage, 1 ㅇ.

\section{Suborder Troctomorpha Family Compsocidae}

7. Compsocus elegans Banks, 1930

Records: A1, 12.XI.2007, canopy fogging, 1 q. Pd, 23.XI.2009, on house wall, 1 q.

Family Amphientomidae

8. Seopsocus sp.

Records: A11, 14.XI. 2007, canopy fogging, 2 ठึ

\section{Family Liposcelididae}

9. Belaphotroctes sp.

Records: FGll, 26.XI.2009, on iron bar, 4 우아.

10. Liposcelis sp.

Record: QC, 29.VI-2.VII.2011, led light trap, 8 우.

\section{Family Pachytroctidae}

Records: S.Ch, 23.XI.2009, beating foliage, 1 ㅇ. S.Ch, 18.X.2010, beating foliage, 2 우오.

\section{Suborder Psocomorpha \\ Family Epipsocidae}

\section{Goja sp.}

Records: S.Ch, 21.XI.2009, beating foli-

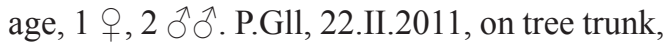
1 q, 2 §ึ. P.Ca, 23.II.2011, on tree trunk, 5 우, 7 ఏ઼.

\section{Mesepipsocus sp.}

Records: Pd, 7.XI.2009, on house wall, 1

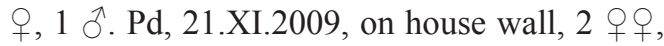

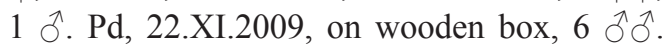
$\mathrm{Pd}$, 24.XI.2009, on palm fronds roof, 1 ․ $\mathrm{Pd}$,

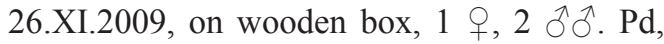
22.XI.2010, on house wall, 3 q,$+ 1 \hat{\jmath}$.

\section{Epipsocus sp. 1}

Record: PB, 19-22.X.2010, Malaise trap, 1 ô. S.PY, 20-22.X.2010, Malaise trap, 1 ठे. PR, 23-25.II.2011, Malaise trap, 1 §ิ. S.PB, 26.II.2011, in leaf litter, 1 ô.

\section{Epipsocus sp. 2}

Record: PB, 19-22.X.2010, Malaise trap, 1 §. CT1, 26.II.2011, led light trap, 1 స.

\section{Family Ptiloneuridae}

16. Loneura gorgonaensis García Aldrete, González \& Sarria, 2011

Records: PP, 24-25.II.2011, led light trap,

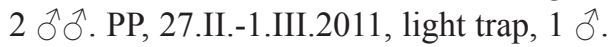

17. Loneura insularis García Aldrete, González \& Sarria, 2011

Records: PP, 22-23.II.2011, led light

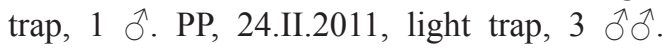
PY, 27.III.2011, led light trap, 1 ऽ. CMr, 28-29.V.2011, led light trap, 1 ऽ̋.

18. Loneura monticola García Aldrete, González \& Sarria, 2011 
Records: CT1, 27.II.-1.III.2011, led light trap, $5 \hat{\jmath} \widehat{\jmath}$. CT, 27.II.-1.III.2011, led light trap, $1 \delta^{\top}$.

\section{Family Cladiopsocidae}

19. Cladiopsocus sp.

Records: A13, 16.XI.2007, canopy fogging, 1 ठ. PB, 19-22.X.2010, Malaise trap,

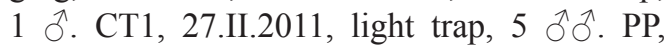
23.II.2011, light trap, 3 $\widehat{\delta} \widehat{\delta}$. S.PY, 23.II.2011, light trap, 1 \%. CMr, 24-25.V.2011, light trap, 2

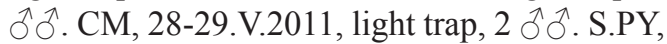
17.II.-2.III.2011, light trap, $1 \widehat{\jmath}$.

\section{Family Dolabellopsocidae}

\section{Dolabellopsocus sp.}

Records: A8, A14, 16.XII.2007, canopy fogging, 3 우. PR, 23-25.II. 2011, Malaise trap, 1 ․ S.PB, 26.II.2011, 1 q. CMr, 24-25.V.2011, led light trap, 1 क; Malaise trap,

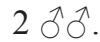

\section{Isthmopsocus sp.}

Records: S.PB, 23-25.II.2011, Malaise trap, 1 ㅇ. S.PB, 26.II.2011, in leaf litter, 3 우우 1 กิ. S.PB, 27.II.2011, in leaf litter, 6 우, 4

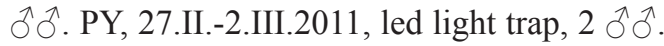
CMr, 24-25.V.2011, led light trap, 4 ถึ ठิ

\section{Family Asiopsocidae}

\section{Notiopsocus $\mathrm{sp}$.}

Records: A14, 16.XI.2007, canopy fogging, 1 ㅇ.

\section{Family Caeciliusidae}

\section{Valenzuela sp. 1}

Records: A1, A3, A10, A15, A16, 12-17. XI.2007, canopy fogging, 3 우, 2 ऽึ. $\mathrm{CMr}$, 24-25.V.2011, led light trap, 3 ठิ ठิ. CT2, 26-27.V. 2011, led light trap, 3 $\widehat{\jmath}$.

\section{Valenzuela sp. 2}

Records: S.Ch, 21 and 23.XI.2009, beating foliage, $2 \widehat{\partial} \widehat{\partial}$. Pd, 24 and 26.XI. 2009, beating foliage, $26 \propto 9$, $17 \hat{\jmath}$. PP, 17.X.2010, beating foliage, 1 o. S.Ch, 18 and 23.XI.2010, beating foliage, 3 우, 1 ํ. S.Ch, 18-22.X.2010, Malaise trap, 1 ふै. S.PB, 19.X.2010, beating foliage, 6 오, 6 구. PB, 19.X.2010, beating foliage, 4 웅, 2 ๙ $\sigma^{\lambda}$. PP, 22.II.2011, beating foliage, 1 J. S.PB, 26 and 28.II.2011, beating foliage, 2 q 9,1 $\hat{\jmath}$. PH, 24.V.2011, beating foliage, 1 . .

\section{Valenzuela sp. 3}

Records: A9, A10, A13, A16, 14.XI.2007,

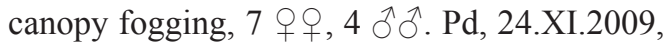
beating foliage, 10 웅, $3 \widehat{\jmath}$. PP, 23.II.2011, beating foliage, 2 웅. S.PB, 28.II.-1.III.2011, light trap, 1 \%. CMr, 24-25.V.2011, light trap, 14 우, 7 ठึ. CM, 28-29.V.2011, light trap, 2 우우. CT1, 26-27.V.2011, light trap, 1 ㅇ․

\section{Valenzuela sp. 4}

Records: A1, 12.XI.2007, canopy fogging, 1 †. A8, 14.XI.2007, canopy fogging, 1 ‥ Pd, 24.XI.2009, beating foliage, 5 우, 1 §. Pd, 26.XI.2009, beating foliage, 1 q. Pd, 22.X.2010, on house wall, 1 §. Acd, 26.II.-1. III.2011, Malaise trap, 1 $\widehat{\delta}$.

27. Valenzuela sp. 5

Records: Pd, 26.XI.2009, beating foliage, 1 ․ PP, 17.X.2010, beating foliage, 1 ㅇ․

\section{Valenzuela sp. 6}

Records: PP, 23.II.2011, beating foliage, 1 ふै. CMr, 24-25.V.2011, led light trap, 1 \%,

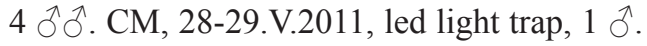

\section{Family Stenopsocidae}

29. Graphopsocus cruciatus (Linnaeus, 1768) Record: A15, 17.XI.2007, canopy fogging, 3 우우, 1 ऊ.

Family Lachesillidae

\section{Lachesilla sp. 1}

Record: S.Ch, 21-22.X.2010, led light trap, 1 J. S.Ch, 23.X.2010, beating foliage, 2 우우, 1 ㄱ. 


\section{Lachesilla sp. 2}

Records: S.PB, 19.X.2010, 2 우, 1 § . PP, 23.II.2011, beating foliage and led light trap, 1 ㅇ, 2 ふิ๋. CT1, 27.II.-1.III.2011, led light trap, 1 ㅇ, 1ठ․ CMr, 24-25.V.2011, led light trap, 1 o. PR, 26.II.2011, beating foliage, 1 q. Acd, 25-26.V.2011, led light trap, 1 J. CT2,

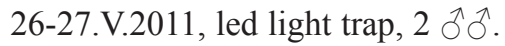

\section{Family Ectopsocidae}

\section{Ectopsocus sp. 1}

Records: PGll, 16.X.2010, beating foliage, 5 웅, 4 ठํ․ S.PB, 25.XI.2009, in leaf litter, 2 우. S.FGll, 26.XI.2009, in leaf litter, 1 ㅇ. PGll, 26.XI.2009, beating foliage, 5 우. PB, 19.X.2010, beating foliage, 1 . .

\section{Ectopsocus sp. 2}

Records: PGll, 26.XI.2009, on palm trunk,

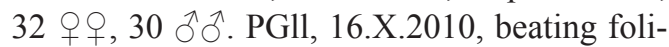
age, 7 우, 7 ठึ.

\section{Ectopsocus sp. 3}

Records: PGll, 26.XI.2009, on palm trunk, 1 ․ Pd, 21.XI.2009, beating foliage, 5 우 . PGll, 16.X.2010, beating foliage, 9 우, 14 ふึે. PCa, 23.II. 2011, beating foliage, 1 ㅇ․

\section{Ectopsocus sp. 4}

Records: PGll, 16.X.2010, beating foliage, 1 స.. S.PB, 19.X.2010, beating foliage, 6 우우, 6 ठิठ

\section{Ectopsocus sp. 5}

Records: Pd, 24.XI.2009, beating foliage, 5 웅, 3 ふึ่̂. PGll, 26.XI.2009, on tree trunk, 1 ‥ PP, 17.X.2010, beating foliage, 2 우, 2 సึ. PB, 19.X.2010, beating foliage, 1 ภ. PP, 24.II.2011, beating foliage, 2 우우.

\section{Ectopsocus sp. 6}

Records: CMr, 24-25.V.2011, led light trap, 10 .

\section{Ectopsocus sp. 7}

Records: PP, 23.II.2011, light trap, 1 q.
39. Ectopsocus sp. 8

Record: S.Ch, 6.VII.2011, on tree trunk with ant's nest, 2 우우.

\section{Family Peripsocidae}

40. Peripsocus sp. 1

Records: A11, 14.XI.2007, canopy fogging, 2 웅․ A16, 17.XI.2007, canopy fogging, 2 ๙ิे. Pd, 22.XI.2009, beating foliage, 5 우오. Pd, 24.XI. 2009, beating foliage, 4 우우. PGll, 26.XI.2009, beating foliage, 1 ․ PGll, 16.X.2010, beating foliage, 1 P. PP, 24.II.2011, led light trap, 1 กิ.

\section{Peripsocus sp. 2}

Records: CMr, 24-25.V.2011, led light

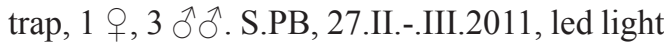
trap, 1 . CT1, 27.II.-2III.2011, led light trap,

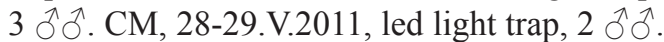

\section{Peripsocus sp. 3}

Records: PP, 23.II.2011, beating foliage,

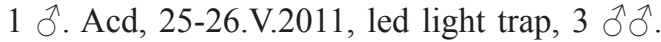
CT1, 27.II.-2.III.2011, led light trap, 2 ठิ $\widehat{~}$. CM, 28-29.V.2011, led light trap, 1 万.

\section{Peripsocus sp. 4}

Records: S.PB, 26.II.2011, beating foliage, 1 +. S.PB, 28.II.2011, beating foliage, $1 \delta^{\lambda}$.

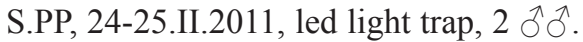

44. Kaestneriella ecuatoriana García Aldrete, 1989

Records: PP, 23.II.2011, beating foliage, 1

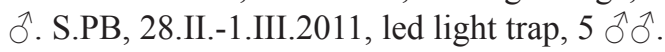
CM, 28-29.V.2011, led light trap, 2 ઠิં

\section{Genus ca. Kaestneriella}

Records: A14, A15, 17.XI.2007, canopy fogging, 2 우. Pd, 24.II.2009, beating foliage, 3 웅․ PGll, 26.XI.2009, beating foliage, 1 ․ PP, 17.X. 2010, beating foliage, 1 ㅇ․ PP, 23.II.2011, beating foliage, 2 웅.

Family Archipsocidae

46. Archipsocus sp. 
Records: A4, 13.XI.2007, canopy fogging, 1 ․ A5, A11, 14.XI.2007, canopy fogging, 2 웅․ A16, 17.XI.2007, canopy fogging, 6 우우. S.Ch, 23.XI. 2009, beating foliage, 2 우우. Pd, 24.XI.2009, beating foliage, 10 우우. PGll, 26.XI.2009, on palm trunk, 2 우. PGll, 16.X.2010, beating foliage, 7 우, 2 추․ PB, 19.X.2010, beating foliage, 1 ․ S.PB. 23.II.2011, in leaf litter, 1 ふ઼. S.PB. 28.II.2011, in beating foliage, 1 ㅇ․

\section{Pararchipsocus sp.}

Records: PGll, 16.X.2010, beating foliage,

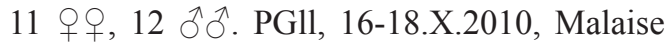
trap, 1 ․ PP, 17.X.2010, beating foliage, 2 우우, 3 ふふふ.

Family Pseudocaeciliidae

\section{Heterocaecilius sp.}

Records: A1, 12.XI.2007, canopy fogging, 1 ㅇ․ Pd, 24.XI.2009, beating foliage, 5 웅․

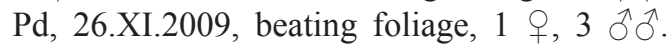
PGll, 26.XI. 2009, beating foliage, 4 웅. PGll, 16.X.2010, beating foliage, 32 오, 17 ठิ $\widehat{\jmath}$. PP,

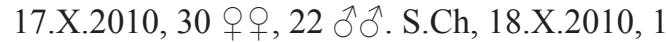

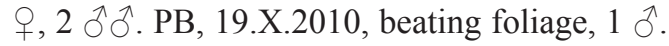
PP, 24.II.2011, beating foliage, $4 \hat{\jmath} \widehat{\jmath}$.

\section{Pseudocaecilius citricola (Ashmead, 1879)}

Records: A1, 12.XI.2007, canopy fogging, 3 우. A10, 14.XI.2007, canopy fogging, 1 ㅇ. Pd, 24.XI.2009, beating foliage, 2 웅․ PGll, 26.XI. 2009, beating foliage, 4 우오. PP, 17.X.2010, beating foliage, 2 우우.

\section{Scytopsocus sp.}

Records: A1, 12.XI.2007, canopy fogging, 4 우. A2, A3, 13.XI.2007, canopy fogging, 5

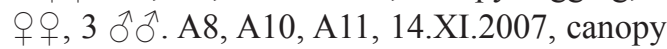
fogging, 2 우, 1 ㄱ. A14, A15,A16, 17.XI.2007,

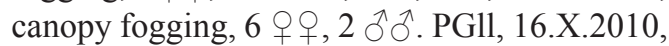
beating foliage, 4 우오. PP, 17.X.2010, beating foliage, 5 웅. S.Ch, 18-19.X.2010, led light trap, 3 กิ่. PB, 19.X.2010, beating foliage, 1 ․ S.Ch, 21-22.X.2010, these, and the following specimens, taken in led light traps, 4
๙ึ. PP, 23.II.2011, 1 ふૈ. S.PP, 24-25.II.2011, 1 ふึ. PY, 27.II.-1.III. 2011, 1 §ิ. CT1, 28.II.-2. III.2011, 1 ઈิ. PY, 28.II.-1.III.2011, 1 ઈे. CMr, 24-25.V. 2011, 9 ठోठ. Acd, 25-26.V.2011, 4

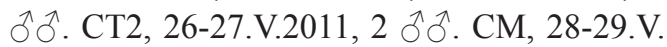
2011, 3 ठิすี.

Family Philotarsidae

\section{Aaroniella sp.}

Records: A3, 13.XI.2007, canopy fogging, 2 우우, 1 ㄱ. A11, 14.XI.2007, canopy fogging, 5 우우. A14, 16.XI.2007, canopy fogging, 1

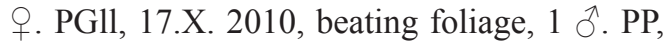
17.X.2010, 1 ㅇ, 1 ठ․ PP, 23.II.2011, light trap, 4 웅, 2 ふึ. PGll, 22.II.2011, beating foliage, 1 ㅇ.

Family Hemipsocidae

52. Hemipsocus africanus Enderlein, 1907

Records: PGll, 26.XI.2009, beating foliage, 18 우, 6 ふึે. PGll, 16.X.2010, beating foliage, 1 ㅇ. PB, 19.X.2010, beating foliage, 10 .

\section{Familia Psocidae}

\section{Blaste sp.}

Records: A11, 14.XI.2007, canopy fogging, 1 ㅇ. A13, 16.XI.2007, canopy fogging, 1 ㅇ․ A15, 17.XI.2007, canopy fogging, 3 우우. PP, 17.X.2010, beating foliage, 3 q 0,1 ๙ิ. PB, 19.X.2010, beating foliage, 3 우, 1 ㄱ. PB, 19-22.X.2010, 1 今. S.Ch, 21-22.X.2010, led light trap, 1 ㅇ.

\section{Blastopsocus sp. 1}

Records: A11, 14.XI.2007, canopy fogging, 1 ㅇ. A15, 17.XI.2007, canopy fogging, 1 ․

\section{Blastopsocus sp. 2}

Records: A4, 13.XI.2007, canopy fogging, 1 ․ A11, 14.XI.2007, canopy fogging, 2 우우. PP, 23.II.2011, led light trap, 1 ㅇ, 1 万.

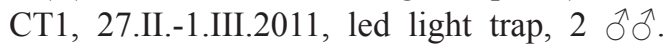


CMr, 24-31.V.2011, Malaise trap, 1 §. CM, 28-29.V.2011, led light trap, 1 ठ.

\section{Blastopsocus sp. 3}

Records: A5, 14.XI.2007, canopy fogging, 1 q. S.Ch, 21-22.X.2010, led light trap, 1 ô. PGll, 22.II.2011, beating foliage, 7 우, 3 $\widehat{\delta}$. CMr, 24-25.V.2011, led light trap, 1 ㅇ.

\section{Blastopsocus $\mathrm{sp} .4$}

Records: PP, 22.II.2011, beating foliage, 1 ‥ PP, 23.II.2011, led light trap, 1 O. CT1, 27.II.2011, led light trap, 2 ठे $\widehat{~}$.

\section{Blastopsocus sp. 5}

Records: A11, 14.XI.2007, canopy fogging, 1 . A14, 17.XI.2007, canopy fogging, 1

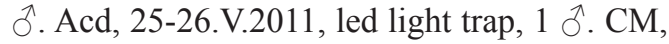

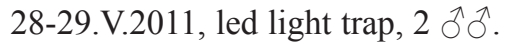

\section{Indiopsocus sp. 1}

Records: A13, 14.XI.2007, canopy fogging, 4 우. A15, 17.XI.2007, canopy fogging, 1 ㅇ.

\section{Indiopsocus sp. 2}

Records: A11, 14.XI.2007, canopy fogging, 1 ․ A15, 17.XI.2007, canopy fogging, 2 ふึ่̂. Pd, 24.XI.2009, beating foliage, 14 우아. PP, 17.X. 2010, beating foliage, 9 웅, 2

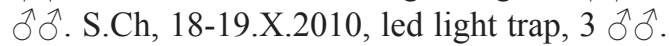
S.Ch, 21-22.X.2010, led light trap, 1 ㅇ, 9 ठิ ठ̂. PB, 19.X.2010, beating foliage, 1 ऽ. S.PY,

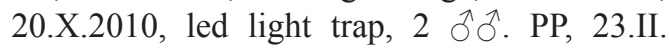
2011, led light trap, 1 ふे. S.PP, 24-25.II.2011, led light trap, 1 O. S.PB, 26.II.2011, beating foliage, 1 ㅇ. PY, 27.II.-1.III.2011, led light

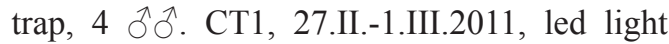
trap, 2 §ิ. S.PB, 28.II.-1.III. 2011, led light trap, 18 ふै. CMr, 24-25.V.2011, led light trap,

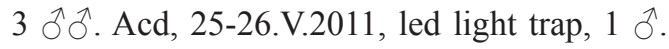
CM, 28-29.V.2011, led light trap, 4 ふึે̂.

\section{Indiopsocus sp. 3}

Record: PB, 19-22.X. 2010, Malaise trap, $1 \delta^{\top}$.

62. Psococerastis sp. 1
Records: A15, A16, 17.XI.2007, canopy fogging, 5 우. S. PB, 24.II.2011, direct capture, 1 ․ S.PY, 27.II.2011, direct capture, 1 ‥ CT1, 27.II.-1.III.2011, led light trap, 3 우, S.PB, 1 ㅇ.

\section{Psococerastis sp. 2}

Records: A15, 17.XI.2007, canopy fogging, 1 J. CT1, 27.II.-1.III.2011, led light trap,

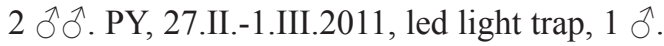

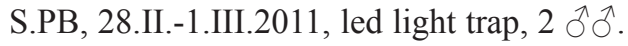

64. Psococerastis sp. 3

Records: S.FGll, 26.XI.2009, beating foliage, 4 우, 1 ○े. PGll, 22.II.2011, in leaf litter, 1 ふ. CMr, 24.V.2011, direct capture, 1 ㅇ․

65. Ptycta sp. 1

Records: A1, 12.XI.2007, canopy fogging, 1 ㄱ. A13, 16.XI.2007, canopy fogging, 4 우우. CMr, 24-25.V.2011, led light trap, 1 ㅇ, 3 ठิ ठิ. CM, 28-29.V.2011, led light trap, 1 ô.

\section{Ptycta sp. 2}

Records: A15, 17.XI.2007, canopy fogging, 1 ô. PP, 23.II.2011, light trap, 1 o, 1

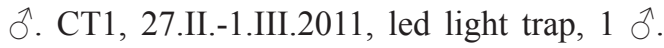

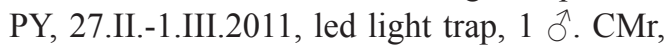
24-25.V.2011, light trap, 5 우, 7 ठึฎ. CM, 28-29.V.2011, led light trap, 3 ふૈ.

\section{Thyrsopsocus sp. 1}

Records: A3, 13.XI.2007, canopy fogging, 1 స. A11, 14.XI.2007, canopy fogging, 1 ․

68. Thyrsopsocus sp. 2

Records: S.Ch, 18.X.2010, beating foliage,

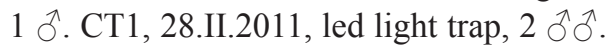

\section{Trichadenotecnum sp.}

Records: A2, 13.XI.2007, canopy fogging, 1 ふึ. CM, 28-29.V.2011, light trap, 1 స.

70. Psocidae New Genus (?)

Records: A8, A11, 14.XI.2007, canopy fogging, 1 ㅇ, 1 ๙. A15, 17.XI.2007, canopy fogging, $1 \hat{\jmath}$. 
Family Myopsocidae

\section{Myopsocus sp. 1}

Records: A2, A3, 13.XI.2007, canopy fogging, 2 우오, 3 ठํ․ A13, 16.XI.2007, canopy fogging, 2 우. A14, A16, 17.XI.2007, canopy fogging, 3 oㅇ. PP, 23.II.2011, these and the following specimens taken in led light traps, 4 우오. PP, 24.II.2011, 1 q. S.PP, 24-25.II.2011, 1 ‥ CT1, 27.II.-1.III.2011, 5 우, 1 §ึ. S.PB, 28.II. 2011, 2 오, 1 ô. CMr, 24-25.V.2011, 2 우우, 2 저. Acd, 25-26.V.2011, 2 우우. PY, 27.II.-1.III.2011, 1 q.

\section{Myopsocus sp. 2}

Records (all taken in led light traps): PP, 23.II.2011, 1 o. CMr, 24-25.V.2011, 6 우우, 3 ふึ่. CM, 28-29.V.2011, 3 우우, 1 ઈิ.

\section{Lichenomima sp. 1}

Records: PB, 19.X.2010, beating foliage, 2 우. PB, 19-22.X.2010, Malaise trap, 1 ㄱ. S.PB, 28.II.2011, led light trap, 1 \%. CMr,

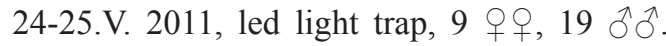
CM, 28-29.V.2011, led light trap, 1 q.

\section{Lichenomima sp. 2}

Records: CMr, 24-25.V.2011, led light trap, 12 오, $41 \hat{\jmath} \hat{\jmath}$. CT2, 26-27.V.2011, led light trap, 1 ㅇ, 1 ก.

\section{Lichenomima sp. 3 \\ Record: CMr, 24-25.V.2011, led light trap, 1 ㅇ.}

\section{DISCUSSION}

The distribution of the abundance of the species found follows the usual pattern of a few species represented by many individuals, many species represented by one or few individuals, and a middle group of species with intermediate values (Table 3). Given the close proximity of Gorgona Island to the mainland, separated by only $35 \mathrm{~km}$, it is not surprising that the species richness be quite high; in comparison with other islands in the Mexican Pacific is meaningful, as it shows that, according to the theory of island biogeography (MacArthur \& Wilson, 1967), small islands have fewer species than large islands, and distant islands have fewer species than close islands to the mainland (Table 4, García-Aldrete, 1986; García Aldrete, Cadena-Carrión \& Cervantes-Peredo, 1992); unfortunately, we still do not have a good psocid census of the mainland near the NNP Gorgona, but the evidence we have indicates that, most probably, the level of endemism is low, so far with only Lepolepis sp., Goja sp., the three species of Loneura, the genus ca. Kaestneriella, and the new genus of Psocidae as probable endemics to NNP Gorgona, so it would seem that the island fauna is an extension of the continental fauna. An interesting feature of the fauna, comparing it with the other Pacific islands, is that only one species, the widely distributed Pseudocaecilius citricola (Ashmead), is shared among the five islands (Table 4).

Of particular interest are the records of Lepidopsocus pretiosus Banks and Nepticulomima hoesemanni Enderlein in the NNP Gorgona; the former was known from Guam, Micronesia, Christmas Island, Indonesia, Melanesia and Polynesia, and the latter was known from several African countries and from Christmas Island, and was recently recorded at the Waorani Ethnic Reserve, in Napo, Ecuador (Lienhard \& Smithers, 2002; García-Aldrete, 2001).

The most abundant species were Echmepteryx madagascariensis (Kolbe), E. falco Badonnel, and Heterocaecilius sp., that together, account for $35.6 \%$ of the specimens collected. The first two are pantropical, and are among the first colonizers of secondary vegetation in the Pacific (Thornton, 1985), which may explain their presence and abundance in the NNP Gorgona. They were found throughout the island, but were most abundant at the beaches and near the small town, where the density of coconut palms is high; this agrees with the finding of Thornton et al. (1988), on Java, Indonesia, where they found lepidopsocids mostly on dead palm fronds.

The diversity of Psocoptera in the biogeographic Chocó is unknown, but upon comparing the genera found in the NNP Gorgona, with 
TABLE 3

Relative abundance of Psocoptera species from the NNP Gorgona (2007-2011)

\begin{tabular}{|c|c|c|}
\hline Species & $\mathrm{N}$ & $\%$ \\
\hline E. madagascariensis & 264 & 15.26 \\
\hline Echmepteryx falco & 229 & 13.24 \\
\hline Heterocaecilius sp. & 123 & 7.11 \\
\hline Indiopsocus sp. 2 & 79 & 4.57 \\
\hline Ectopsocus sp. 2 & 76 & 4.39 \\
\hline Valenzuela sp. 2 & 74 & 4.28 \\
\hline Nepticulomima hoesemanni, Scytopsocus sp. & 63 & 3.64 \\
\hline Lichenomima sp. 2 & 55 & 3.18 \\
\hline Valenzuela sp. 3 & 51 & 2.95 \\
\hline Archipsocus sp. & 35 & 2.02 \\
\hline Lichenomima sp. 1 & 33 & 1.91 \\
\hline Myopsocus sp. 1 & 32 & 1.85 \\
\hline Ectopsocus sp. 3 & 30 & 1.73 \\
\hline Pararchipsocus sp. & 29 & 1.68 \\
\hline Hemipsocus africanus & 26 & 1.50 \\
\hline Isthmopsocus $\mathrm{n} . \mathrm{sp}$. & 21 & 1.21 \\
\hline Ptycta sp. 2 & 20 & 1.16 \\
\hline Mesepipsocus sp. (n.sp.), Aaroniella sp. & 19 & 1.10 \\
\hline Goja sp. (n. sp.), Ectopsocus sp. 1 & 18 & 1.04 \\
\hline Lepidopsocus pretiosus & 17 & 0.98 \\
\hline Cladiopsocus sp., Ectopsocus sp. 5, Peripsocus sp. 1 & 16 & 0.92 \\
\hline Blaste sp. & 15 & 0.87 \\
\hline Myopsocus sp. 2 & 14 & 0.81 \\
\hline Lachesilla sp. 2, Ectopsocus sp. 4, Blastopsocus sp. 3 & 13 & 0.75 \\
\hline Pseudocaecilius citricola & 12 & 0.69 \\
\hline Valenzuela sp. 1, Valenzuela sp. 4, Psococerastis sp. 1, Peripsocus sp. 2 & 11 & 0.64 \\
\hline Peripsocus sp. 2, Ptycta sp. 1 & 10 & 0.58 \\
\hline ca. Kaestneriella sp. (n. gen.), Blastopsocus sp. 2 & 9 & 0.52 \\
\hline Liposcelis sp., Dolabellopsocus sp., Kaestneriella ecuatoriana & 8 & 0.46 \\
\hline Valenzuela sp. 6, Peripsocus sp. 3, Psococerastis sp. 3 & 7 & 0.40 \\
\hline Loneura insularis, Loneura monticola, Psococerastis sp. 2 & 6 & 0.35 \\
\hline Blastopsocus sp. 5, Indiopsocus sp. 1 & 5 & 0.29 \\
\hline $\begin{array}{l}\text { Lepolepis sp., Belaphotroctes sp., Epipsocus sp. 1, Graphopsocus cruciatus, Lachesilla sp. 1, } \\
\text { Peripsocus sp. 4, Blastopsocus sp. } 4\end{array}$ & 4 & 0.23 \\
\hline Proentomum personatum, Tapinella sp., Loneura gorgonaensis, Thyrsopsocus sp. 2, New Genus. & 3 & 0.17 \\
\hline $\begin{array}{l}\text { Seopsocus sp., Epipsocus sp. 2, Valenzuela sp. 5, Ectopsocus sp. 8, Compsocus elegans, Blastopsocus } \\
\text { sp. 1, Thyrsopsocus sp. 1, Trichadenotecnum sp. }\end{array}$ & 2 & 0.12 \\
\hline Notiopsocus sp., Ectopsocus sp. 6, Ectopsocus sp. 7, Indiopsocus sp. 3, Lichenomima sp. 3 & 1 & 0.06 \\
\hline
\end{tabular}

those recorded in Colombia, we found an index of faunistic similarity of $42 \%$, meaningful but preliminary, and likely to go down, as the Colombian fauna gets to be better known. Also, upon comparing Fisher's $\alpha$ diversity index for the psocids of the NNP Gorgona, with the values for other areas (Table 5), such as Fortuna,
Panama, and the Krakatau archipelago, with areas of 195 and $25.4 \mathrm{Km}^{2}$ respectively, we found a relatively high value, given the small area of the NNP Gorgona $\left(13.8 \mathrm{Km}^{2}\right)$.

In Colombia, 21 families, 42 genera and 87 species have been recorded (Lienhard \& Smithers, 2002; García-Aldrete et al., 2011a; 
TABLE 4

Number of species of Psocoptera (S), area $\left(\mathrm{Km}^{2}\right)$, and distance to mainland (DM, Km) in five Pacific islands

\begin{tabular}{lccc}
$\quad$ Island & $\mathrm{S}$ & Area $\left(\mathrm{Km}^{2}\right)$ & $\mathrm{DM}(\mathrm{Km})$ \\
María Madre & 48 & 144 & 128 \\
San Juanito & 16 & 8 & 152 \\
Socorro & 19 & 167 & 704 \\
Clarión & 6 & 25 & 1076 \\
Gorgona Island & 75 & 13.8 & 35 \\
\hline
\end{tabular}

TABLE 5

Richness of Psocoptera and relation to area

\begin{tabular}{lcc}
\multicolumn{1}{c}{ Locality } & Area $\left(\mathrm{Km}^{2}\right)$ & $\alpha$ de Fisher \\
Barro Colorado (Panamá) (Broadhead \& Wolda, 1985) & 15.64 & 24.6 \\
Chamela (Jalisco, México) (García Aldrete, 1988) & 500 & 24.01 \\
Fortuna (Panama) (Broadhead \& Wolda, 1985) & 195 & 14.8 \\
Krakatoa Islands (Indonesia) (Thornton et al., 1988) & 25.4 & 16.7 \\
Los Tuxtlas (Veracruz, México) (García Aldrete et al., 1997) & 150 & 32.45 \\
NNP Gorgona (this study) & 13.8 & 15.9 \\
\hline
\end{tabular}

García-Aldrete, González \& Sarria-S, 2011; González et al., 2011). Accordingly, families Asiopsocidae, Compsocidae, Hemipsocidae, Myopsocidae and Stenopsocidae constitute new records for the country, raising to 26 the number of families for the country. The genera Cladiopsocus, Echmepteryx, Epipsocus, Graphopsocus, Hemipsocus, Heterocaecilius, Kaestneriella, Lepidopsocus, Lichenomima, Mesepipsocus, Myopsocus, Nepticulomima, Notiopsocus, Pararchipsocus, Proentomum, Ptycta, Scytopsocus, Seopsocus, Thyrsopsocus and Trichadenotecnum raise to 62 the number of genera for the country; in addition, we found two genera and ten species new to science.

In summary, the psocid fauna of the NNP Gorgona requires further study, to identify to species level, and to verify which of the species are undescribed. The rich psocid fauna of the NNP Gorgona, as compared to the psocid fauna of other Pacific islands, particularly the Mexican Tres Marías and Revillagigedo archipelagos, and the closeness to the continent, indicates that its fauna is an extension of the continental fauna. To ascertain the point above requires a psocid survey of the continental fauna in the area closest to the NNP Gorgona.

\section{ACKNOWLEDGMENTS}

FSS and RGO are grateful to the Departamento de Biología, Universidad del Valle, for research support. ANGA thanks Instituto de Biología, Universidad Nacional Autónoma de México for research support over the years. We also wish to thank Andrew Mason and Fernando Montealegre-Z., for allowing us to study the material collected by them during the development of their project in the NNP Gorgona (research permit No. DTSO-G-31); we are grateful to the Administration and personnel of the NNP Gorgona Island, and to Alan Giraldo, head of the Project "Evaluation of the present status of the subjects of faunistic conservation in Gorgona Island" (research permit No. PIBD DTSO 011-10). Julián Alexander Mendívil, Diana Marcela Torres Domínguez and Stephany Valdés-R., students of the Biology Department, Facultad de Ciencias Naturales y Exactas, Universidad del Valle, are especially 
thanked for their invaluable help and logistic support during field work in Gorgona Island.

\section{RESUMEN}

La fauna de Psocoptera (Psocodea) del Parque Nacional Natural Gorgona, consiste de 75 especies en 42 géneros y 21 familias. 1730 especimenes fueron recolectados en el periodo entre noviembre de 2007 y junio de 2011. Cinco familias, 20 géneros y nueve especies son nuevos registros para Colombia, y dos géneros y diez especies son nuevos para la ciencia. La fauna de Psocoptera de la isla constituye una extensión de la fauna continental.

Palabras clave: Costa del Pacífico, Colombia, los parques nacionales naturales, la lista de especies.

\section{REFERENCES}

Badonnel, A. (1951). Ordre des Psocoptères. In P. P. Grassé (Ed.), Traité de Zoologie (pp. 1301-1340). Paris, France: Masson et Cie.

Bess, E., Smith, V., Lienhard, C. \& Johnson, K. P. (2006). Psocodea. Parasitic lice (=Phthiraptera), Book Lice, and Bark Lice. Retrieved from http://tolweb.org/

Chamorro, C. (1990). Suelos. In C. Aguirre \& J. O. Rangel (Eds.), Biota y ecosistemas de Gorgona (pp. 65-72). Fondo FEN, Bogotá, Colombia.

García-Aldrete, A. N. (1986). Especies de Psocoptera (Insecta), de las Islas María Madre y San Juanito, Nayarit, México. Acta Zoológica Mexicana, 13: 1-29.

García-Aldrete, A. N. (1988). The psocids (Psocoptera) of Chamela, Jalisco, México. Species, diversity, abundance distribution and seasonal changes. Folia entomológica mexicana, 77: 63-84.

García-Aldrete, A. N. (1990). Insecta: Psocoptera. In D. L. Dindal (Ed.), Soil Biology Guide (pp. 1033-1052). John Wiley \& Sons. Inc., USA.

García-Aldrete, A. N., Cadena-Carrión, A. \& CervantesPeredo, L. (1992). Psocoptera (Insecta) of Socorro and Clarión Islands, Revillagigedo Archipelago, Mexico. Species and comparisons with the continental and the Tres Marías Archipelago psocid faunas. Acta Zoológica Mexicana, 50: 1-20.

García-Aldrete, A. N. (2001). Remarkable records of Psocoptera in the neotropics. Biogeographica, 77 (3): 123-128.

García-Aldrete, A. N. (2006). New genera of Psocoptera (Insecta), from México, Belize and Ecuador
(Psoquillidae, Ptiloneuridae, Lachesillidae). Zootaxa, 1319: 1-14.

García-Aldrete, A. N. (2008). Psocópteros (Insectos). In S. Ocegueda \& J. Llorente-Bousquets (Coords.), Catálogo taxonómico de especies de México, capital Natural de México (CD 1). Vol. I: Conocimiento actual de la biodiversidad. México: CONABIO.

García-Aldrete, A. N. \& Mockford, E. L. (2009). A list of Psocoptera (Insecta: Psocodea) from Brazil. Revista Mexicana de Biodiversidad, 80: 665-673.

García-Aldrete, A. N., González R. \& Sarria, F. A. (2011). Three new species of Loneura (Psocodea: "Psocoptera": Ptiloneuridae) from Gorgona Island, Cauca, Colombia, with a new infrageneric classification. Zootaxa, 3050: 55-62.

González-Obando, R., García-Aldrete, A. N. \& Carrejo, N. S. (2011). A new species of Steleops Enderlein, and a Colombian record of $S$. pulcher New (Psocodea: "Psocoptera": Psocidae). Zootaxa, 2735: 23-27.

Lienhard, C. (1998). Psocoptères euro-méditerranéens. Faune de France 83. Paris: Fédération Française des Sociétés de Sciences Naturelles.

Lienhard, C. \& Smithers, C. N. (2002). Classification of Psocoptera (with checklist of genera). Retrieved from: http://www.ville-ge.ch/musinfo/mhng/page-e/ ps-clas.htm

Macarthur, R. H. \& Wilson, E. O. (1967). The Theory of Island Biogeography. Princeton: University Press Princeton.

Mockford, E. L. (1993). North American Psocoptera (Insecta). Flora and Fauna Handbook No. 10. Gainesville, Florida: Sandhill Crane Press.

New, T. R. (1987.) Biology of the Psocoptera. Oriental Insects, 21: 1-109.

Rangel, O. \& Rudas, A. (1990). Aspectos microclimáticos. In J. Aguirre \& O. Rangel (Eds.). Biota y Ecosistemas de Gorgona. (pp. 41-51). Fondo FEN, Bogotá, Colombia.

Simberloff, D. S. \& Wilson, E. O. (1969). Experimental zoogeography of islands: The colonization of empty islands. Ecology, 50: 278-296.

Thornton, I. W. B. (1985). The geographical and ecological distribution of arboreal Psocoptera. Annual Review of Entomology, 30: 175-196.

Thornton, I. W. B., New, T. R. \& Vaughan, P. J. (1988). Colonization of the Krakatau Islands by Psocoptera (Insecta). Philosophical Transactions of the Royal Society Biological Sciences, 322 (1211): 427-443. 